\title{
Creating a Virtual Chatbot to Scaffold Skills DEVELOPMENT IN FIRST-YEAR ENGINEERING EDUCATION
}

\author{
Ilya Kreynin, MohammedShabbar Manek, Chirag Variawa \\ University of Toronto \\ ilya.kreynin@mail.utoronto.ca,m.manek@mail.utoronto.ca,chirag.variawa@utoronto.ca
}

\begin{abstract}
A habit is a consistent repetition of a behaviour in a stable context, making the behaviour automatic over time. Strong study and wellness habits, such as reflective journaling, predict academic success in undergraduate students. Scalable digital solutions could support positive habit formation in first year undergraduate engineering students. To test this idea, four versions of an SMS chatbot that enables reflective journaling via text message were developed. One version acted as a control, and the other three each implemented a habit driver - reminders to journal, positive reinforcement upon journal completion, and automatic reporting of journaling adherence to an anonymous partner (social proof). Students interested in reflective journaling $(N=28)$ used the chatbot for 28 days. Study results showed that positive reinforcement had no noticeable effect, reminders improved journaling adherence but reduced habit formation, and social proof improved both adherence and habit formation. These results indicate that chatbots can be effective, accessible, and scalable tools in scaffolding positive habit development in first year undergraduate engineering students.
\end{abstract}

Keywords: Habits, Reflective Journaling, Chatbots, Automaticity, First Year Undergraduate Engineering Education

\section{INTRODUCTION}

This section details the motivation behind this paper, poses the research question, gives a background on habit science and habit drivers, and explains the choice of focus on journaling and SMS chatbots.

\subsection{Motivation}

Habit formation is an important part of long-term behavioural change, and is especially important in early post-secondary education [1]. Well known education habits such as note taking [2] and class attendance [3], are strong predictors of academic success. However, strong wellness-oriented habits, such as consistency and amount of sleep [4], consistent exercise [5], meditation [6], and journaling [7] have also been shown to not only improve student health and well-being, but also significantly improve academic performance.

The increased academic demands and novel context of engineering education often demand the formation of new habits. This is because high stress environments, such as engineering education, increase the rate of habitual behaviour [8]. First-year engineering students may have formed some of these positive habits prior to starting university. However, because habits are situational [9] and often change in novel environments, these prior habits may not persist through the transition into postsecondary education.

Thus, the habits of first year engineering students are at risk at a time when they are most important. Given the benefits of both wellness and education habits for university student well-being and academic performance, engineering educators should encourage the formation of positive habits. The guiding question behind this paper is: can technology be used to scaffold habit formation in first year engineering students?

\subsection{A Brief Primer on Habits}

A habit is defined as a consistent repetition of a behaviour in the presence of stable contextual clues that increase the automaticity of said behaviour [9]. One key difference between habits and other behaviours is their high automaticity. Automaticity is the ability to perform a behaviour with minimal cognitive load. Habits are behaviours with high automaticity, meaning that they occur with little conscious intent.

In the context of behavioural consistency, automaticity is related to, but distinct from, adherence. Adherence is simply the consistency with which a behaviour is repeated. Adherence is distinct from automaticity, as a behaviour could conceivably be repeated on a regular basis while having low automaticity. Both are important to measure when studying and evaluation habit formation [10].

Along with high automaticity, stable contextual clues distinguish habits from other behaviours. Habits have a specific structure that promotes automaticity. Environmental cues, otherwise known as triggers or 
trigger events, are vital for habit formation [11] Conscious association of behaviours with triggers, often existing elements in an individual's routine have been shown to increase automaticity.

\subsection{Habit Drivers}

While triggers are vital to the development of habits, other potential habit drivers have been shown to increase automaticity of behaviour over time. Positive reinforcement after the completion of the desired behaviour $[12,13]$, reminders to complete the behaviour at the intended time [14], and the social pressure [15] of having adherence levels regularly reported to an outside source have all been shown to improve habit formation in certain environments. Due to their backing in prior literature and ease of implementation, positive reinforcement, reminders, and social proof are explored as potential drivers of automaticity increase in first year engineering students.

\subsection{Supporting Habits Through Technology}

The study of habit-supporting technology is becoming of increasing importance within the field of humancomputer interaction [13]. While many pieces of technology label themselves as assisting with habit formation, very few are grounded in research, mostly focusing on self-tracking and reminders [10]. There is a lack of technology that scaffolds behaviours with the structure required for habit formation [10].

Despite this, the opportunity to use smartphone applications and other easily distributed technology for habit formation is clear. Their presence and consistent use in everyday life gives them the potential to be a powerful delivery vehicle for triggers and other habit drivers [16, 17]. Due to their novel and high-stress circumstances, first year engineering students could especially benefit from habit formation assistance from smartphone applications.

\subsection{Why Journaling?}

The habit selected for study was reflective journaling, due to its wide-ranging benefits for engineering students and the ease of adherence tracking for research purposes.

Reflective journaling is the practice of regularly recording one's thoughts, opinions, or feelings about recent and/or upcoming events. Journaling has many cognitive and psychological benefits, and has been shown to increase self-efficacy [18], improve academic performance [7], and reduce anxiety [19] in students. As self-efficacy and anxiety have strong relationships with academic performance in first year engineering, along with clear benefits to well-being, journaling is both an academic and well-being habit. A journaling habit would therefore have wide-ranging benefits for first year engineering students.
One key reason journaling was selected as the habit to study is the ease with which adherence can be verified. When journaling is performed digitally, as it is in this study, verifying and recording whether or not a participant has journaled can be made accurate automatic without additional action or breach of privacy. This allows for a small research team to study a large population.

Due to time and resource constraints, journaling was the only habit studied. Future studies applying the same method to other relevant habits should be conducted.

\subsection{Why SMS Chatbot?}

This study was conducted using a chatbot-style interface, due to ease of use, high accessibility, ease of development, and ease of data collection.

A chatbot is a computer program that conducts a conversation with a user via text. Chatbots have existed since the 1960s, and have been used for a wide variety of use cases, including information retrieval, learning a new language and personal assistants [20]. Today, all major modern messaging applications support chatbots, including SMS, Facebook Messenger, Telegram and WeChat.

Chatbots have one key advantage for the user - low information overload. This translates to fewer distractions and less information to attend to in a delivery method a user is comfortable with [21]. In the high stress environment of first year engineering, students do not have the luxury of time. This lightweight delivery method would be optimal to help them get into the habit of reflective journaling.

While chatbots are supported across a wide variety of messaging platforms, this study was conducted using an SMS chatbot. This is because all phones support SMS, without reliance on the download of an additional application or consistent internet access. This high accessibility allows the highest possible number of students to participate in the study.

Another reason for the selection of a chatbot interface was the ease and flexibility of development. The team was able to rapidly and cheaply create a chatbot application called the Reflective Journaling Bot that trains participants in its use, enables digital reflective journaling, and records adherence without violating the privacy of participants. These advantages allow small research teams, like the one behind this study, to develop and distribute chatbot applications for studies with large sample sizes.

Finally, a chatbot-style interface allowed the research team to easily and anonymously collect data. Anonymity is important, because reflective journaling can often involve the recording of intimate or personal information. The chatbot interface allows for the anonymous collection of phone numbers without association with a name. It also allows the team to record adherence (whether or not a 
participant journaled on a given day) without accessing or storing the contents of their journal entry.

The team measured automaticity via the Self-Report Behavioural Automaticity Index (SRBAI), which is a short questionnaire. The distribution of the SRBAI could also be done easily and anonymously via the SMS interface.

\section{PROCEDURE AND METHODOLOGY}

This section of the report details the procedure followed in this study, introduces the Reflective Journal Bot (RJB), and expands on ethical considerations.

\subsection{Procedure Overview}

Below is a high level overview of the procedure followed for this study - each step is expanded on in further detail.

1. Develop the RJB tool

2. Recruit first year engineering students interested in reflective journaling $(\mathrm{N}=28)$ to participate in the study, anonymously collecting their phone numbers

3. Split participants into 4 groups by habit driver

4. Launch, monitor, and end study over a 28 day period

5. Measure adherence and automaticity of participants Habit formation is an important part of long-term

\subsection{Reflective Journal Bot}

To conduct this investigation, a software tool called the Reflective Journal Bot (RJB) was developed. This tool is an SMS-based chatbot with five main functions:

1. Gather consent for study participation

2. Explain the purpose of and procedure for the study being conducted

3. Enable users to journal by texting RJB, scaffolding their journaling with 3 specific questions

4. Implement habit drivers - reminders, positive reinforcement, and social proof - through contextspecific text messages

5. Distribute information, such as the SRBAI survey, and any administrative updates from the research team

All interactions between a user and RJB occur within the SMS interface on their phone. To start using RJB, a user sends a text message to RJB's phone number. RJB then explains the study and acquire the user's consent to continue via a "Yes" or "No" message. After consent is acquired, a user can journal any time by sending any message to RJB. RJB takes them through a three question reflective journaling process, in which RJB asks questions to prompt the user to reflect on their day and participants journal by responding to those questions via text message.
After the three questions are answered, RJB acknowledges receiving them and ends the journaling session.

\subsection{Ethical Considerations}

This study was designed with three core ethical considerations in mind: anonymity, privacy and voluntary participation.

Anonymity: To start journaling, participants simply need to send a text message to RJB. No other information is required of the participants.

Privacy: Reflective journaling is a personal and private activity, and RJB keeps it that way. None of the text from journal entries was recorded or saved in RJB's database, so the research team has no way to access the content of journal entries.

Voluntary Participation: Participation in this study is $100 \%$ voluntary, and participants could opt-out at any time by texting "STOP" to RJB. This ceases all communications from RJB and deletes the user's phone number and data from the database. No participants chose to use this option.

All of the above information was presented to participants prior to signing up, and again at the start of the study.

\subsection{Participant Recruitment}

Participants $(\mathrm{N}=28)$ were recruited from a population of first year undergraduate engineering students at the University of Toronto. Participants all had a prior interest in developing the habit of reflective journaling, with 12 of the 28 having tried and failed to establish a journaling habit in the past. Critically, no participant had ever journaled regularly (more than once every 2 weeks) prior to participating in the study. No external compensation, other than the opportunity to potentially develop a journaling habit, was given.

Participants were told they would be expected to journal using RJB shortly before going to bed every day for 28 days.

To preserve participant anonymity, and reduce the researcher team's ability to link a journaling pattern to a specific participant, participants were recruited in 2 stages. First, participants signed up via an online form that explained the purpose of the study and their scope of responsibility. This form collected participant name and email, but did not ask for a phone number. Once recruitment was completed, all participants were sent an email asking them to text RJB on the study's start date to begin journaling. This process removed the link between a participant and their phone number, preserving their anonymity. This study was designed with three core ethical considerations in mind: anonymity, privacy and voluntary participation. 


\subsection{Grouping by Habit Driver}

To test the effectiveness of different habit drivers, participants were randomly split into 4 groups. Three of these groups interacted with version of RJB modified to implement a specific habit driver - positive reinforcement, reminders, and social proof - and one was used as a control.

The positive reinforcement group received encouraging messages after completing a journaling session. The reminder group receive reminders at $8: 30 \mathrm{pm}$ night reminding them to journal. Participants in the social proof group were anonymously paired up with another participant, and received a message every morning indicating whether or not their partner had completed their journaling task the previous night - they were also told their adherence was being anonymously reported in the same way.

\subsection{Data Collection}

The results were primarily analyzed using two metrics, adherence and automaticity, across the four experimental groups, one for each habit driver.

Adherence was measured as the proportion of days on which the participant completed their journal over the course of the study. Automaticity was measured by distributing a digital version of the Self-Reported Behavioural Automaticity Index, or the SRBAI [22]. This is a scientifically valid four-question subset of the broader Self-Reported Habit Index (SRHI), and measures automaticity. The SRBAI was administered via an anonymous online form sent through a text message in RJB, and was used to mark the end of the study.

\section{RESULTS}

This section of the report details quantitative findings from the study, specifically the levels of adherence and automaticity observed across various habit drivers. Each experimental group had 7 participants.

\subsection{Adherence}

Adherence was measured as the proportion of study days on which a journal was completed.

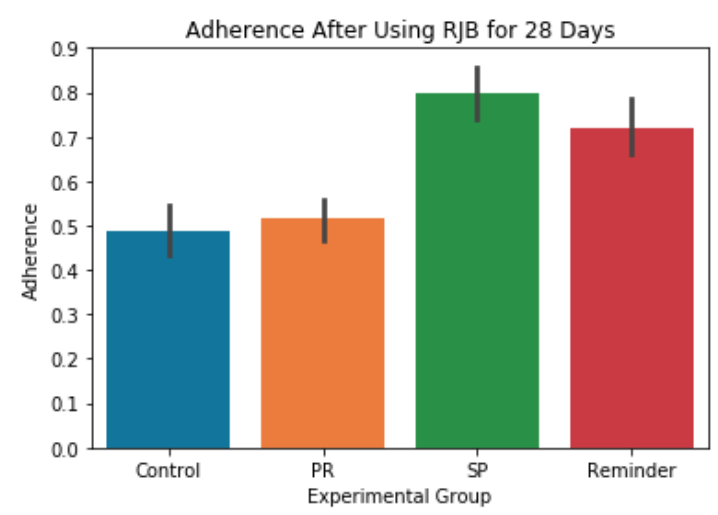

Fig. 1. RJB average adherence after 28 days of use across selected habit drivers.

The social proof (mean $=0.77, \mathrm{SD}=0.10$ ) and reminder (mean $=0.75, \mathrm{SD}=0.07$ ) groups performed significantly better in terms of adherence than the control (mean $=0.47$, $\mathrm{SD}=0.11$ ) and positive reinforcement (mean $=0.51$, $\mathrm{SD}=0.10$ ) groups.

\subsection{Automaticity}

Automaticity was measured via an online SRBAI questionnaire distributed at the end of the study. The SRBAI consists of four questions, each answered on a scale from 1 (Strongly Disagree) to 7 (Strongly Agree) - a 4 represents the neutral (Neither Agree Nor Disagree) response. The highest possible score on the SRBAI is therefore 28, and the lowest possible score is 4 .

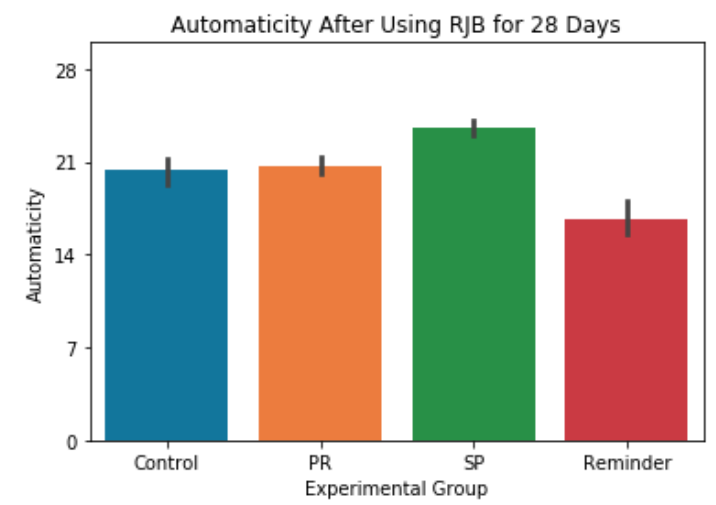

Fig. 2. RJB average automaticity after 28 days of use across selected habit drivers.

The social proof group (mean=20.8, SD=1.37) had the highest automaticity, while the reminder group (mean=16.67, SD=1.75) had the lowest. The control (mean=20.8, SD=1.37) and positive reinforcement (mean=20.67, $\mathrm{SD}=0.84$ ) groups. 
Table 1: RJB average adherence and automaticity after 28 days of use across selected habit drivers.

\begin{tabular}{|c|r|r|r|r|}
\hline & \multicolumn{2}{|c|}{ Adherence } & \multicolumn{2}{c|}{ Automaticity } \\
\hline & Mean & \multicolumn{1}{c|}{ SD } & Mean & \multicolumn{1}{c|}{ SD } \\
\hline Control & 0.47 & 0.11 & 20.80 & 1.37 \\
\hline PR & 0.51 & 0.12 & 20.67 & 0.82 \\
\hline SP & 0.77 & 0.10 & 23.50 & 0.84 \\
\hline Reminder & 0.76 & 0.07 & 16.67 & 1.75 \\
\hline
\end{tabular}

\section{DISCUSSION}

This section of the report discusses the significance of the results, comments on their potential applications to engineering education, acknowledges the limitations and assumptions made in this study, and identifies areas for future work.

\subsection{General Discussion}

From prior research on the effectiveness of SMS messaging in the formation of consistent behaviours, it was expected that reminder group would have higher adherence and lower automaticity than the control group [10]. This was supported by the results of the study, which indicate that while reminders are useful for remembering to journal, they actually hinder the formation of the journaling habit. This is important in a first year engineering context, as high stress environments elicit habitual behaviours. A lack of automaticity could therefore lead to reduced adherence in times of high stress, which are often when positive habits are needed most for sustained academic performance and well-being.

The strong results of the social proof group, which had the highest performance in both adherence and automaticity, is promising. Developing a habit without sacrificing adherence in the short term represents the best of both worlds, and these results would serve engineering students well if applied to study and wellness habits. This is especially promising when considering the scalability of the social proof method, as it was effective despite the complete anonymity of both participants in all social proof pairings - this indicates that social proof is an effective habit driver beyond an individual's social circles, allowing this technique to be applied among groups of relative strangers, such as new classes of first year engineering students.

Given that both social proof and positive reinforcement were administered via text message, the contrast in their effectiveness is interesting. Both have been shown to improve adherence when administered by a live partner in person $[12,13,15]$, but differed widely in the study. As discussed previously, social proof outperformed control by a wide margin, while positive reinforcement had no significant additional effect. This may be due to a low quality or variety of positive reinforcement reward messages, or it may indicate that positive reinforcement is indeed only effective when administered in person.
RJB's general success in scaffolding a journaling habit is also promising, especially given that 12 of the 28 participants had previously tried and failed to form a journaling habit. The reminder and social proof groups both had adherence levels of over 0.76 ; this is the rough equivalent of journaling on all weekdays and taking weekends off. This is a high level of adherence for a new behaviour, indicating that the use of chatbots to scaffold other beneficial habits in first year engineering students deserves further study.

\subsection{Limitations}

While the research team was happy to find 28 students interested in participating in this study, future studies should be conducted with a larger number of participants.

The average time taken to form a habit is 66 days [13], though this time can be lower if the action is simple. The research team hoped that journaling via text message into RJB would be a sufficiently simple behaviour to observe habit formation over a shorter 28 day timespan. High automaticity scores for non-reminder groups indicate that this occurred, but future work should nevertheless validate study results over a longer period of time.

\subsection{Future Work}

While the results of this study are promising, further research is required to validate the effectiveness of SMS chatbots in scaffolding habit formation. Among variables to explore are different participant populations, different wellness and study habits, and different habit drivers.

\section{CONCLUSION}

The guiding question behind this paper was whether or not technology could be used to scaffold habit formation in first year engineering students. Through the development of a simple SMS chatbot tool to assist students with the formation of a reflective journaling habit, the research team was able to improve both the adherence and automaticity of a journaling behaviour, and identified social proof as the most effective habit driver in this context.

These findings could enable engineering educators to use the broad scalability and accessibility of digital technology to support wellness and academic habits in first year engineering students. If successful, such an effort would improve student well-being and academic performance, making further research and investment into habit supporting technology a worthwhile pursuit.

\section{References}

[1] S. A. Nonis and G. I. Hudson, "Performance of College Students: Impact of Study Time and Study Habits," Journal of Education for Business, vol. 85, no. 4, pp. 229$238,2010$. 
[2] Yu and D. D., "How Much Do Study Habits, Skills, and Attitudes Affect Student Performance in Introductory College Accounting Courses?.," New Horizons in Education, 30-Nov-2011. [Online]. Available: https://eric.ed.gov/?id=EJ955535.

[3] M. Credé, S. G. Roch, and U. M. Kieszczynka, "Class Attendance in College," Review of Educational Research, vol. 80, no. 2, pp. 272-295, 2010.

[4] M. T. Trockel, M. D. Barnes, and D. L. Egget, "HealthRelated Variables and Academic Performance Among First-Year College Students: Implications for Sleep and Other Behaviors," Journal of American College Health, vol. 49, no. 3, pp. 125-131, 2000.

[5] D. Bellar, L. Judge, J. Petersen, A. Bellar, and C. Bryan, "Exercise and academic performance among nursing and kinesiology students at US colleges," Journal of Education and Health Promotion, vol. 3, no. 1, p. 9, 2014.

[6] D. Bellar, L. Judge, J. Petersen, A. Bellar, and C. Bryan, "Exercise and academic performance among nursing and kinesiology students at US colleges," Journal of Education and Health Promotion, vol. 3, no. 1, p. 9, 2014.

[7] M. D. N. Lew and H. G. Schmidt, "Self-reflection and academic performance: is there a relationship?," Advances in Health Sciences Education, vol. 16, no. 4, pp. 529-545, 2011

[8] L. Schwabe and O. T. Wolf, "Stress Prompts Habit Behavior in Humans," Journal of Neuroscience, vol. 29, no. 22, pp. 7191-7198, 2009.

[9] P. Lally and B. Gardner, "Promoting habit formation," Health Psychology Review, vol. 7, no. sup1, 2013.

[10] K. Stawarz, A. L. Cox, and A. Blandford, "Beyond SelfTracking and Reminders," Proceedings of the 33rd Annual ACM Conference on Human Factors in Computing Systems - CHI 15, 2015.

[11] P. M. Gollwitzer, "Implementation intentions: Strong effects of simple plans.," American Psychologist, vol. 54, no. 7, pp. 493-503, 1999.

[12] H. Aarts, T. Paulussen, and H. Schaalma, "Physical exercise habit: on the conceptualization and formation of habitual health behaviours," Health Education Research, vol. 12, no. 3, pp. 363-374, 1997.
[13] P. Lally, C. H. M. V. Jaarsveld, H. W. W. Potts, and J. Wardle, "How are habits formed: Modelling habit formation in the real world," European Journal of Social Psychology, vol. 40, no. 6, pp. 998-1009, 2009.

[14] K. Stawarz, A. L. Cox, and A. Blandford, "Dont forget your pill!," Proceedings of the 32nd annual ACM conference on Human factors in computing systems - CHI 14, 2014.

[15] P. Gellert, J. P. Ziegelmann, L. M. Warner, and R. Schwarzer, "Physical activity intervention in older adults: does a participating partner make a difference?," European Journal of Ageing, vol. 8, no. 3, pp. 211-219, 2011.

[16] T. D. Aungst, "Smartphone medication adherence apps: Potential benefits to patients and providers: Response to Dayer et al.," Journal of the American Pharmacists Association, vol. 53, no. 4, pp. 344-345, 2013.

[17] C. Free, G. Phillips, L. Watson, L. Galli, L. Felix, P. Edwards, V. Patel, and A. Haines, "The Effectiveness of Mobile-Health Technologies to Improve Health Care Service Delivery Processes: A Systematic Review and Meta-Analysis," PLoS Medicine, vol. 10, no. 1, 2013.

[18] Fritson, K. K., \& Mandernach, B. J. (2009). K. K. Fritson and B. J. Mandernach, "Impact of Journaling on Students Self-Efficacy \& Locus of Control," PsycEXTRA Dataset, 2009.

[19] Sgoutas-Emch, S.A., \& Johnson, C.J, (1998). Is Journaling Writing an Effective Method of Reducing Anxiety Towards Statistics?

[20] Shawar, Bayan \& Atwell, Eric. (2007). Chatbots: Are they Really Useful?. LDV Forum. 22. 29-49.

[21] "The User Experience of Chatbots," Nielsen Norman Group. [Online]. Available: https://www.nngroup.com/articles/chatbots/. [Accessed: 29-Apr-2019].

[22] B. Gardner, C. Abraham, P. Lally, and G.-J. D. Bruijn, "Towards parsimony in habit measurement: Testing the convergent and predictive validity of an automaticity subscale of the Self-Report Habit Index," International Journal of Behavioral Nutrition and Physical Activity, vol. 9, no. 1, p. 102, 2012. 